\title{
PREDICTION OF AN ICEBERG DRIFT TRAJECTORY DURING A STORM
}

\author{
by \\ D. S. Sodhi and Mona El-Tahan \\ (Faculty of Engineering and Applied Science, Memorial University of Newfoundland, St. John's, \\ Newfoundland, A1B 3×5, Canada)
}

\begin{abstract}
A numerical model is presented for predicting iceberg drift trajectories from known or derived information regarding iceberg characteristics and the environmental forces affecting the motion of an iceberg. The validity of such a model is studied by comparing predicted and observed trajectories of icebergs near Saglek, Labrador, during a storm on 21-22 August 1972 . At this time, iceberg positions (determined by radar), winds, and currents were monitored as part of an oceanographic study, conducted by the Faculty of Engineering and Applied Science,

Memorial University of Newfoundland. The comparison between observed and predicted iceberg drift trajectories is good when the characteristics of the iceberg are assumed to be those of a mediumsized non-tabular iceberg. In order to appreciate the effect of wind and current forces on the drift of the iceberg, several trajectories are plotted in which various environmental forces are excluded. From this study, it is evident that a good prediction of an iceberg drift trajectory is only possible if rather detailed information is available on the current and wind field.
\end{abstract}

LIST OF SYMBOLS

$A_{\text {a }}$ cross-section area of iceberg perpendicular to wind direction in above-water portion

$A_{j} \quad$ cross-section area of iceberg perpendicular to relative current direction in $j$ th 1 ayer

$C_{\mathrm{dw}}, C_{\mathrm{da}}$ drag coefficient of iceberg in water and air, respectively

f $\quad 2 \Omega \sin \phi$, Coriolis parameter

M total mass of iceberg plus added mass

$M_{j} \quad$ mass of water displaced by iceberg in $j^{\text {th }} 1$ ayer

$n$ number of layers in water column $(n=2$, in this paper)
$S_{j} \quad \sqrt{\left(U_{j}-u\right)^{2}+\left(V_{j}-v\right)^{2}}$, relative speed of current with respect to the iceberg in $j$ th layer

$t \quad$ time

$u, v \quad$ components of iceberg velocity

$U_{g}, V_{g} \quad$ components of measured current at $75 \mathrm{~m}$ depth

$U_{j}, V_{j} \quad$ components of current velocity in $j^{\text {th }}$ layer

$U_{\mathrm{S}}, V_{\mathrm{S}} \quad$ components of measured current at $13 \mathrm{~m}$ depth

$U_{w}, V_{w} \quad$ wind-generated currents

$U_{1}, V_{1} \quad$ average velocity component in boundary layer

$V_{0} \quad$ current speed at surface

W $\quad$ wind speed

$x, y$ position of iceberg with respect to a coordinate system in which $x$ and $y$ axes are in the directions of east and north, respectively

z depth below water surface

$\alpha_{j}, \beta_{j}$ components of water acceleration computed from changes in $U_{j}, V_{j}$

$\Omega \quad$ angular speed of Earth $\left(\mathrm{rads}^{-1}\right)$

$\phi \quad$ latitude of location

$\rho_{w}, \rho_{a}$ density of water and air, respectively

$\theta$ direction towards which wind blows, measured anti-clockwise from $x$-axis

INTRODUCTION

Renewed interest in the detailed movements of icebergs began with the advent of oil exploration on the continental shelf off eastern Canada, where their occurrence presents considerable hazards to drilling operations and shipping. 
On the other hand, icebergs may possibly be a source of fresh water if they can be towed from the Antarctic to arid parts of the world.

Whether an iceberg is to be used or avoided, it is important to learn about its drift under the influence of environmental forces such as winds and currents.

In this paper, an iceberg drift model is presented which considers the motion of an iceberg due to wind and water drag forces, the Coriolis force, the pressure-gradient force resulting from the sea-surface slope, and the water acceleration. The validity of this model can only be studied by comparing predicted and observed iceberg drift trajectories when data on wind and currents are collected simultaneously and used as input to the model.

Such an opportunity presented itself during a storm on 21-22 August 1972, near Saglek, Labrador, where the Memorial University of Newfoundland (MUN) was conducting an oceanographic study in collaboration with the Bedford Institute of Oceanography (BIO). During the study, iceberg positions were monitored with the help of a land-based radar at Saglek, while current and wind data were gathered by CSS Dowson, provided by BIO. The predicted iceberg drift trajectory was close to that observed for a specific iceberg in this area. A detailed account of activities during this study is given by Allen (1972).

\section{THE ICEBERG DRIFT MODEL}

Iceberg motion is the net result of forces which vary with time and space. Some of these forces are due to gravity, pressure gradient, wind drag, water drag, Coriolis effects, waves, and swells. Since this study is mainly concerned with the horizontal movement of icebergs, only the horizontal components of these forces are considered. Wave and swell forces are neglected in this study as their magnitudes in the horizontal directions are unknown in comparison to other forces. The mathematical model takes into account the significant environmental forces due to water drag, wind drag, Coriolis acceleration, and sea-surface slope (pressure gradient).

The drag force due to the water is proportional to the square of the relative velocity of water with respect to the iceberg. The constant of proportionality depends upon the size and shape of the under-water portion of the iceberg. Ocean currents represent a complicated mixture of different types of aperiodic and periodic water movements, a few of which are the geostrophic current, the wind-driven current, the inertia current, and the tidal current. The distribution of the magnitude and direction of the various current components varies with time, depth, and location. For the purpose of modelling iceberg drift, the water column is divided into two layers, and the water drag force is then obtained as the vectorial sum of the drag forces which are expressed in terms of relative velocity of the current with respect to the iceberg in each layer.

Similarly, the magnitude and direction of the wind drag force depends on the size and shape of the above-water portion of the iceberg. The average ratio of the iceberg velocity to wind speed is about 0.03-0.04 (Murray 1969); thus in the expression for wind drag force, the relative velocity of wind with respect to the iceberg is taken to be the wind velocity itself.

The Coriolis force, due to the rotating

frame of reference with the Earth, tends to move the iceberg and the water surrounding the iceberg to the right of their path (clockwise) in the northern hemisphere. In a geostrophic current, the pressure-gradient force on an iceberg due to a sloping sea surface balances the Coriolis force due to its movement. If the iceberg motion is not along a geostrophic current direction, there are two forces acting on the iceberg: the Coriolis force due to its movement and the pressure gradient due to the sea-surface slope. In the present study, the pressure-gradient force in each layer is expressed as the negative of the current's Coriolis force (Neumann 1968, Mountain 1980). Thus the combined action of the Coriolis force and the pressure-gradient force on the iceberg is equivalent to expressing the Coriolis force of the iceberg in terms of the relative velocity of the iceberg with respect to the farfield current in a particular layer.

If the water around the iceberg is accelerating due to some potential field, the same potential field would also be acting on the iceberg to accelerate it. So the momentum equation must include a term which takes into account the forces accelerating the water mass and the iceberg at the same time, and this force on the iceberg will be equal to the product of mass of the iceberg and the acceleration of water surrounding the iceberg. This was. first pointed out by Bayly (1971) in a discussion of a paper by Cochkanoff and others (1971) (also see Napoleoni, unpublished). The concept of added water mass is introduced to account for inertial drag due to the acceleration of the water around an object, and the object behaves as if a mass were added to it. The added mass can be determined from the potential flow theory, and it has been assumed to be equal to half of the mass of the iceberg.

The equations of motion taking all the above-mentioned forces into account are written below in the component form:

$$
\begin{aligned}
& \frac{\mathrm{d} x}{\mathrm{~d} t}=u, \\
& \frac{\mathrm{d} y}{\mathrm{~d} t}=v, \\
& \frac{\mathrm{d} u}{\mathrm{~d} t}=\frac{1}{M}\left[\sum _ { j = 1 } ^ { n } \left\{\frac{1}{2} C_{\mathrm{dw}} \rho_{\mathrm{w}}^{A} j\left(U_{j}-u\right) S_{j}+M_{j} \alpha_{j}+\right.\right. \\
& \left.\left.+M_{j} f\left(v-V_{j}\right)\right\}+\frac{1}{2} C_{\mathrm{da}}{ }^{\rho} \mathrm{a}^{A} \mathrm{a}^{W^{2}} \cos \theta\right], \\
& \frac{\mathrm{d} v}{\mathrm{~d} t}=\frac{1}{M}\left[\sum _ { j = 1 } ^ { n } \left\{\frac{1}{2} C_{\mathrm{d} w^{\rho}{ }{ }^{A} j}\left(V_{j}-v\right) S_{j}+M_{j}{ }^{\beta} j\right.\right. \\
& \left.\left.-M_{j} f\left(u-U_{j}\right)\right\}+\frac{1}{2} C_{\mathrm{da}}{ }^{\rho} \mathrm{A}^{A} \mathrm{a} W^{2} \sin \theta\right],
\end{aligned}
$$

where the symbols used are defined above. If the parameters related to the iceberg are known, the above set of equations may be integrated to obtain the response of the model in the form of the iceberg velocity and position, provided the current and wind data are supplied 
as the forcing function (or input to the model). Since the set of differential equations are coupled and nonlinear, it is expeditious to integrate them with the help of a digital computer, and the fourth-order Runge Kutta method is employed for this purpose.

\section{THE ICEBERG PARAMETERS}

Since the mass, area, and drag coefficients of the icebergs under study are not known, these values are chosen from the range quoted in the literature. Thus, the cross-sectional area and mass data for the iceberg under study are given the values shown in Table 1 , which was compiled by the U.S. Coast Guard from observations made over a number of years to establish seven classes of iceberg (Mountain 1980), and the values of the drag coefficients in water and air are assumed to be 1 and 1.5 , respectively, regardless of the size and shape of the iceberg.

A more detailed discussion of this can be found in E1-Tahan (1980).

\section{INPUT TO THE MODEL}

\section{Current data}

As mentioned above, the icebergs were tracked by radar at Saglek while the currents and wind speeds were recorded simultaneously by current meters and C S S Dawson, respectively. Figure 1 shows the trajectories of five icebergs and the locations of the current meters A,B,C and D. At each of these four locations, current meters were installed at depths of $13 \mathrm{~m} \mathrm{(A1,B1,}$ $\mathrm{C} 1, \mathrm{D} 1)$ and $75 \mathrm{~m}(\mathrm{~A} 2, \mathrm{~B} 2, \mathrm{C} 2, \mathrm{D} 2)$ below the sea surface, and three additional meters were installed at locations A, B, and C at depths of $165 \mathrm{~m}$ (A3), $146 \mathrm{~m} \mathrm{(B3),} \mathrm{and} 176 \mathrm{~m}$ (C3), respectively. The data obtained from these current meters were presented and analysed by Holden (1974), who concluded that the current near the surface oscillated with the tidal period of $12.5 \mathrm{~h}$ before the storm of 22 August 1972 and with the inertial oscillation period after the storm.

We shall pay attention to the iceberg drift trajectory 20C because it is close to the current meters at locations A and B. Since the iceberg drift model needs the average water velocity components in the two layers, the oceanic boundary

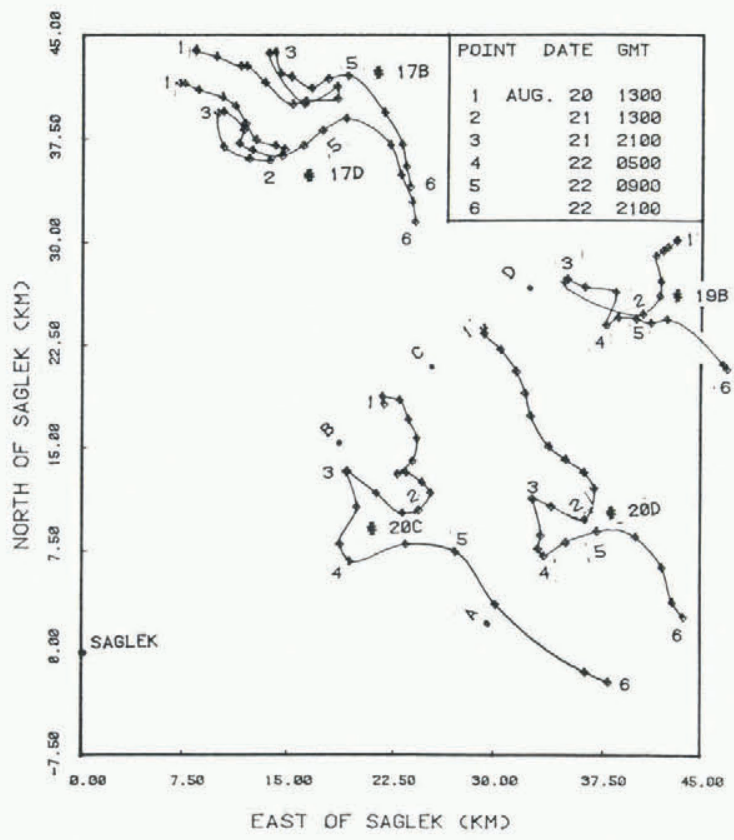

Fig.1. Drift pattern of icebergs offshore Saglek after the storm in August 1972, showing locations of current meters.

layer $\left(U_{1}, V_{1}\right)$ and the deeper water below $\left(U_{2}, V_{2}\right)$, the following procedure is adopted to obtain the average velocities from the data of the current meters. The average current velocity of the deeper layer is taken to be equal to the current velocities measured by current meters at $75 \mathrm{~m}$ depth. All the current meters at $75 \mathrm{~m}$ depth recorded only the direction of the current and not the magnitude due to malfunction, and hence the magnitudes measured by current meters $\mathrm{A} 3$ and B3 at deeper depths are assumed to be the same as those at $75 \mathrm{~m}$ depth. This is justified because the geostrophic current magnitude is more or less constant below the oceanic boundary layer. Let us designate the components of the measured

TABLE I. ICEBERG CHARACTERISTICS (Mountain 1980 with acknowledgement to R.Q. Robe)

Size Mass $\left(10^{6} \mathrm{~kg}\right) \quad$ Dry area $\left(\mathrm{m}^{2}\right) \quad$ Wet area $\left(\mathrm{m}^{2}\right)$ per depth 1 ayer

$0-20 \mathrm{~m} \quad 20-50 \mathrm{~m} \quad 50-100 \mathrm{~m} \quad 100-120 \mathrm{~m}$

Non-tabular icebergs

\begin{tabular}{|c|c|c|c|c|c|c|}
\hline Growler & 0.45 & 10 & 80 & 0 & 0 & 0 \\
\hline Sma11 & 75 & 230 & 780 & 820 & 0 & 0 \\
\hline Medium & 900 & 910 & 1800 & 1900 & 2700 & 0 \\
\hline Large & 5500 & 2000 & 3500 & 3750 & 5300 & 1400 \\
\hline
\end{tabular}

Tabular icebergs

\begin{tabular}{|c|c|c|c|c|c|c|}
\hline Sma 11 & 245 & 650 & 1900 & 2600 & 0 & 0 \\
\hline Medium & 2170 & 2700 & 4400 & 5900 & 8700 & 0 \\
\hline Large & 8235 & 5200 & 7200 & 9700 & 14400 & 5000 \\
\hline
\end{tabular}


current at $75 \mathrm{~m}$ depth to be $U_{\mathrm{g}}$ and $V_{\mathrm{g}}$, and the corresponding components at $13 \mathrm{~m}$ depth to be $U_{\mathrm{S}}$ and $V_{\mathrm{S}}$. From the above discussion, we have

$$
U_{2}=U_{g}
$$

and

$$
V_{2}=V_{g}
$$

To calculate the average velocity component $\left(U_{1}, V_{1}\right)$ in the boundary layer, we assume that the wind-generated currents $\left(U_{W}, V_{w}\right)$ have the distribution with respect to depth in the form given by Ekman (Neumann 1968). If the wind shearstress vector is acting in the positive $y$ direction (i.e. to the north), the wind-generated velocity components are given below:

$$
U_{\mathrm{W}}=V_{\mathrm{o}} \exp (-\pi z / D) \cos (\pi / 4-\pi z / D),
$$

and

$$
V_{\mathrm{w}}=V_{\mathrm{o}} \exp (-\pi z / \mathrm{D}) \sin (\pi / 4-\pi z / D)
$$

where

$$
D \text { is } 36.7 / \sqrt{\sin \phi}(\mathrm{m}) \text {. }
$$

The latitude of Saglek is $58^{\circ} 9^{\prime} \mathrm{N}$. and the depth of the boundary layer $D$ is calculated to be $39.7 \mathrm{~m}$ which is approximately equal to the depth of the mixing layer as is evident from the contour lines of measured salinity-temperaturedepth data (Allen 1972). The net water mass transport, $S_{x}$ and $S_{y}$, in the easterly and northerly directions are given by Neumann (1968) as:

$$
S_{x}=\rho_{\mathrm{w}} \frac{V_{\mathrm{o}} D}{\pi \sqrt{2}}
$$

and

$$
S_{y}=0 \text {. }
$$

This is a remarkable result for it shows that the total water-mass transport is to the right of wind shear-stress direction in the northern hemisphere and to the left in the southern hemisphere. The net effect of wind-driven currents on the motion of an iceberg is to integrate the drag forces at different levels in the boundary layer. In this paper, it is assumed that the boundary layer has a uniform velocity such that the net water-mass transport due to wind effects is equal to that given by Equations 9 and 10, and the average velocity components are $\bar{U}_{w}=0.225 V_{0}$ and $\bar{V}_{W}=0$. Using Equations 7 and 8 , the velocity components at $13 \mathrm{~m}$ depth are $U_{W}=0.358 V_{0} \cos$ $\left(-13.9^{\circ}\right)$ and $V_{W}=0.358 V_{O} \sin \left(-13.9^{\circ}\right)$. Hence, we obtain a factor, equal to 0.628 , which is the ratio of the magnitude of the average velocity in the boundary layer to that at $13 \mathrm{~m} \mathrm{depth}$, and the difference in their directions is $13.9^{\circ}$ (clockwise). On the basis of this discussion, the average water velocity components $\left(U_{1}, V_{1}\right)$ in the boundary layer are obtained by the following expression which effectively subtracts the geostrophic current from the surface current, rotates the resultant by $13.9^{\circ}$ in the anti-clockwise direction, multiplies it by a factor of 0.628 , and adds the geostrophic velocity components.

$$
\begin{aligned}
& U_{1}=0.628\left[\left(U_{s}-U_{g}\right) \cos \left(13.9^{\circ}\right)-\right. \\
& \left.-\left(V_{s}-V_{g}\right) \sin \left(13.9^{\circ}\right)\right]+U_{g},
\end{aligned}
$$

and

$$
\begin{aligned}
& V_{1}=0.628\left[\left(U_{\mathrm{s}}-U_{\mathrm{g}}\right) \sin \left(13.9^{\circ}\right)+\right. \\
& \left.+\left(V_{\mathrm{s}}-V_{\mathrm{g}}\right) \cos \left(13.9^{\circ}\right)\right]+V_{\mathrm{g}} .
\end{aligned}
$$

Since the current velocity varies spatially as well as temporally, the current data near the iceberg are obtained by interpolating the current values at corresponding depths at locations $A$ and B. A cubic interpolation function is used to give more weight to the current meter closer to the iceberg.

Wind data

The surface wind velocity and direction were obtained from the $\log$ books of C S S Dawson which was in the area gathering other oceanographical and meteorological data.

\section{RESULTS AND DISCUSSION}

As mentioned previously, the mass and cross-section areas of the iceberg under study are not known, and the values of these parameters are assumed to be those given in Table 1. Figures 2 and 3 show the predicted trajectories of sma11, medium, and large non-tabular icebergs along with the observed trajectory of iceberg $20 \mathrm{C}$ during the storm. The predicted trajectory of a medium non-tabular iceberg (Fig. 2) is close to the observed trajectory, and thus iceberg $20 \mathrm{C}$ is assumed to be a medium non-tabular iceberg, an assumption also supported by field observations (personal communication from R.T. Dempster).

Figure 4 shows two predicted trajectories when the iceberg is driven by the wind alone and by the currents alone. Figure 5 shows two predicted trajectories when the iceberg is driven by geostrophic currents only and by geostrophic currents and wind together. In these two figures, the effect of excluding a particular environmental force can be seen as the observed trajec-

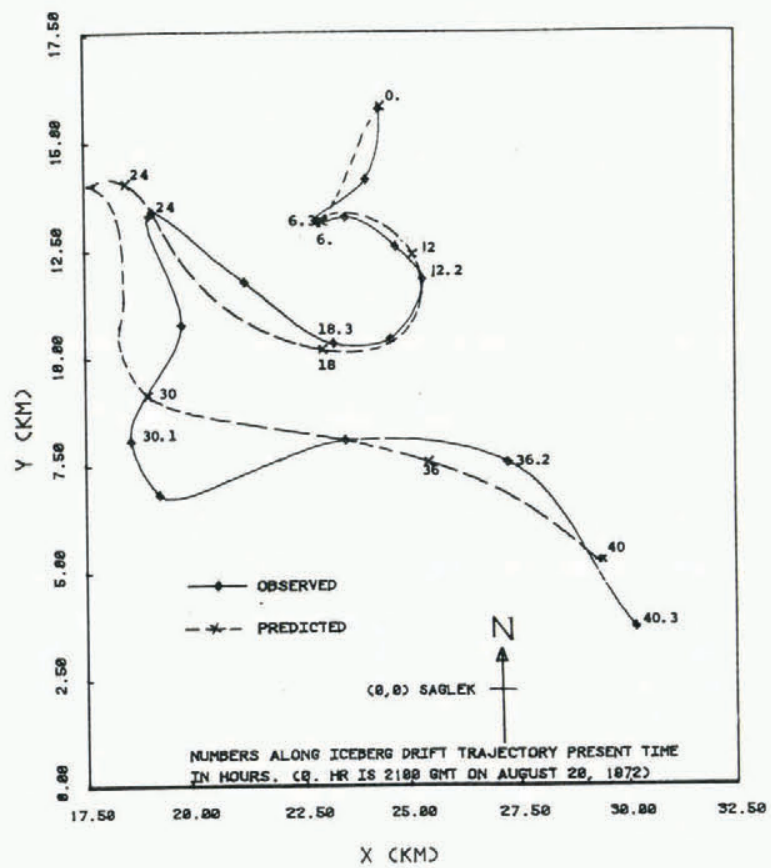

Fig. 2. Predicted and observed trajectories of iceberg 20C during the storm which passed over the Labrador Sea on 21-22 August 1972. 
tory is also plotted there. It is evident from these results that currents and wind have a significant effect on iceberg drift.

Figure 6 shows the effect of including and excluding the Coriolis force on the predicted

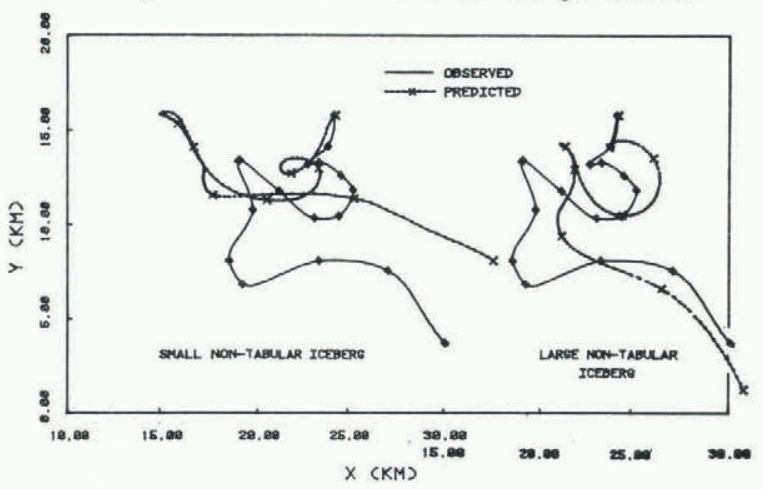

Fig.3. Effect of iceberg size on the predicted trajectory.

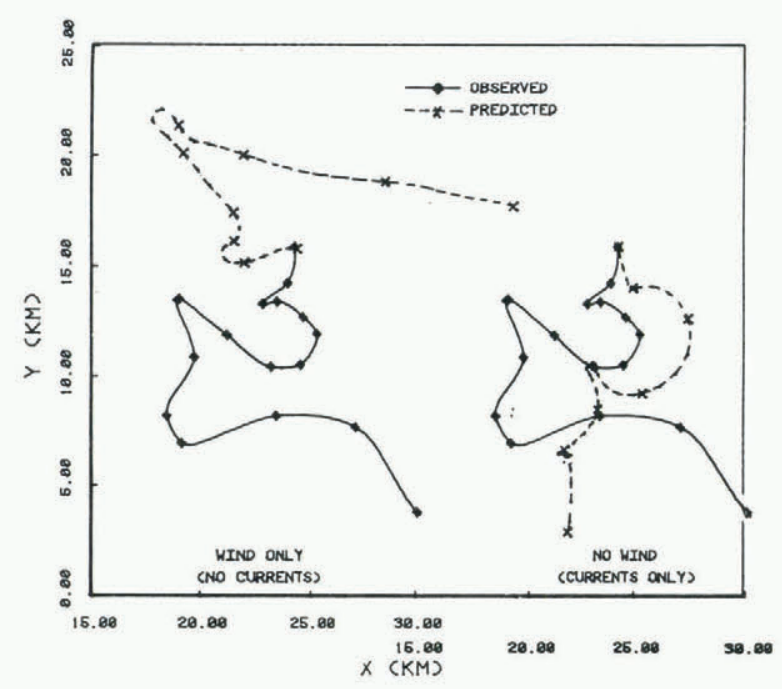

Fig.4. Effect of wind forces on iceberg drift.

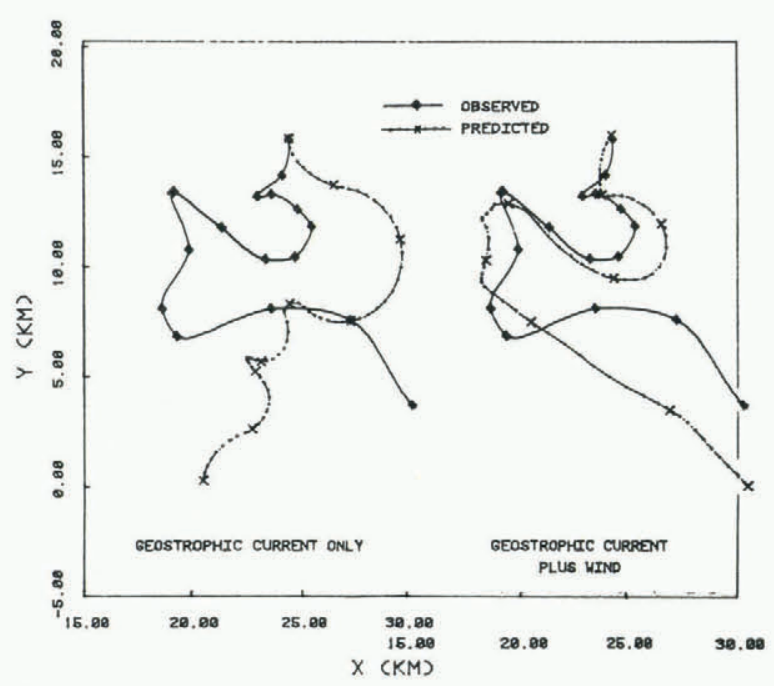

Fig.5. Iceberg drift in geostrophic current with inclusion and exclusion of wind. iceberg drift trajectory. There is a considerable difference in the trajectories, and hence inclusion of Coriolis force is essential for a good prediction of trajectories. Figure 7 depicts the effect of including and excluding the water-acceleration term in the drift model. Though the net effect of excluding this term is not large, a better correlation is obtained in this case by including it in the model. Finally, Figure 8 shows the difference between the trajectories when current data at $13 \mathrm{~m}$ depth are used for the average current velocity in the boundary layer as opposed to using Equations 11 and 12. As is evident from the results, the current data at $13 \mathrm{~m}$ depth as input to the model do not give a good prediction of the iceberg drift, and these data along with the data at greater depths have to be used to estimate the wind-generated currents in the boundary layer.

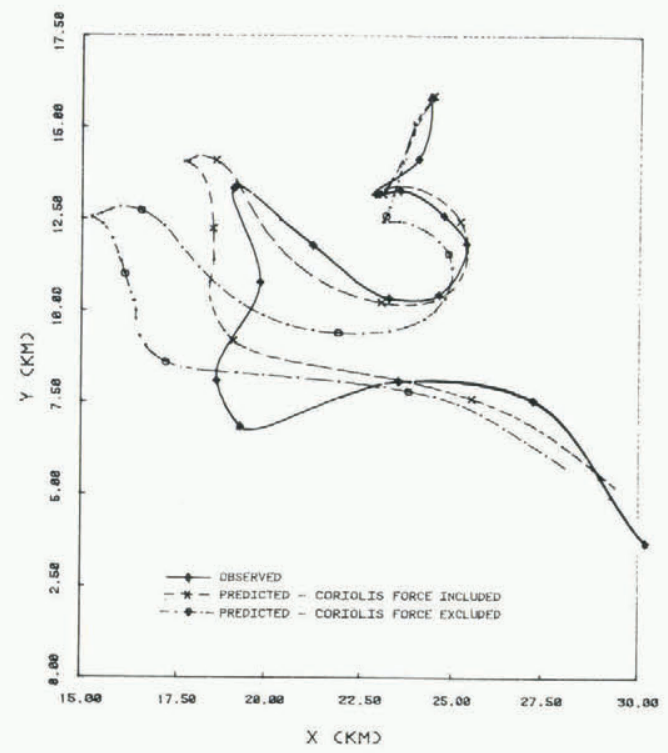

Fig.6. Effect of Coriolis force on iceberg drift.

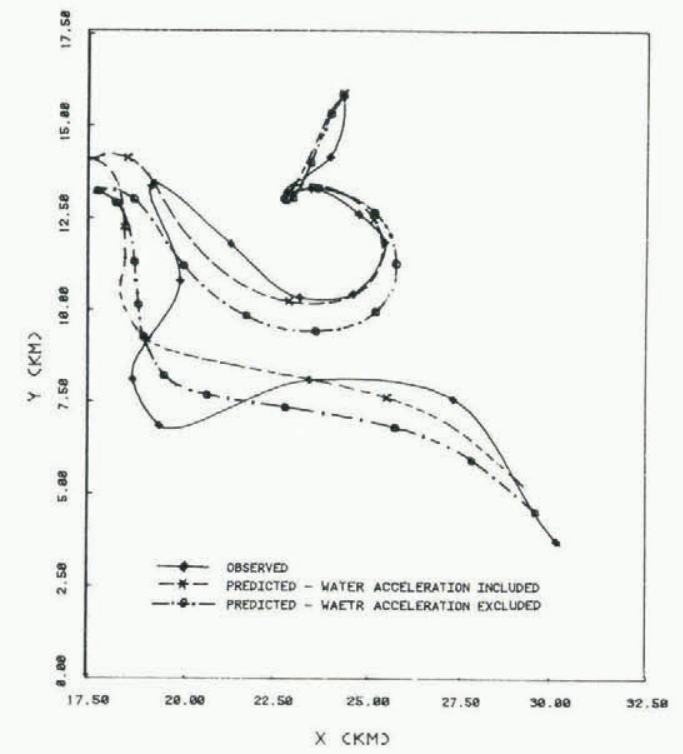

Fig.7. Predicted iceberg drift with inclusion and exclusion of water acceleration. 


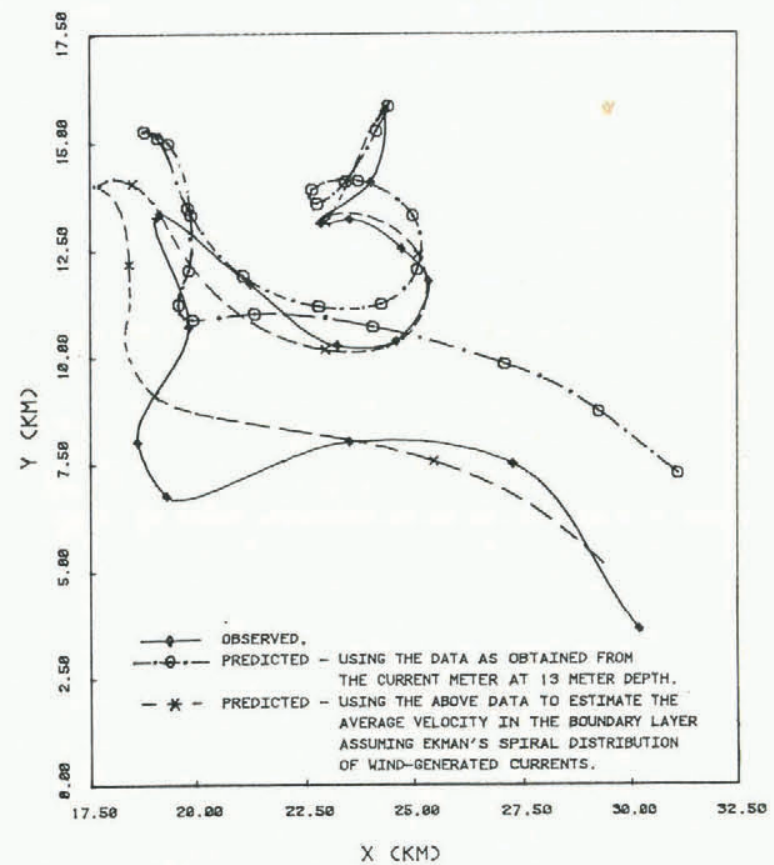

Fig.8. Effect of considering the distribution of the wind-generated current velocity.

\section{CONCLUSIONS}

A numerical model for the iceberg drift is presented, and the model is used to predict the drift trajectory of an iceberg using current and wind data from the field. The trajectory of only one iceberg is predicted because this particular iceberg $(20 \mathrm{C})$ drifted close to the installed current meters. The predicted trajectory compares well with the observed trajectory when the iceberg parameters are assumed to be those of a medium non-tabular iceberg (Table I). The trajectories of other icebergs are disturbed by the storm in a similar manner as shown in Figure 1, and the deviations from the regular drift trajectories start and stop at about the same time. The direct action of the wind is evident from the fact that the icebergs are moved back and forth as the wind direction changes due to the passage of the storm centre through the area. The indirect action of wind on the drift of icebergs is important because of the windgenerated currents and inertial oscillations in the boundary layer.

In this paper, we have shown that the physics of the iceberg drift model are known to the extent that a good prediction of an iceberg trajectory during a storm can be made provided detailed current and wind data are available as input to the model.

\section{ACKNOWLEDGEMENTS}

The authors are grateful to Dean R.T. Dempster, Faculty of Engineering and Applied Science, Memorial University of Newfoundland, St. John's, Newfoundland, for making available the iceberg data and to Dr Miles McPhee of the U.S. Army Cold Regions Research and Engineering Laboratory, Hanover, N.H., for his discussion. The help of many others is also gratefully acknowledged in gathering all the data which led to successful completion of this work.
REFERENCES

Allen J H and others 1973 Iceberg study, Saglek, Labrador, including cruise report C.S.S. Dawson, August 7 to August 26, 1972. Memorial University of Newfoundland. Faculty of Engineering and Applied Science. Report

Bayly I M 1971 Contribution on the inclusion of certain terms in the equations used in the simulation for the prediction of ice movement by Dr. 0. Cochkanoff et a1. In Proceedings of the Canadian seminar on icebergs, Halifax, Nova Scotia, 1971. Ottawa, Dept of National Defence: 153155

Cochkanoff O, Graham J W, Warner J L 1971 Simulation techniques in the prediction of iceberg motion. In Proceedings of the Canadian seminar on icebergs, Halifax, Nova Scotia, 1971. Ottawa, Dept of Nationa1 Defence: 135-152

E1-Tahan M Unpublished Modelling of iceberg drift. M.Eng. thesis, Memorial University of Newfoundland, Faculty of Engineering and Applied Science, 1980

Holden B J Unpublished Some observations on the Labrador Current at Saglek, Labrador. M.Eng. thesis, Memorial University of Newfoundland, Faculty of Engineering and Applied Science, 1974

Mountain D 1980 On predicting iceberg drift. Cold Regions Science and Technology $1(3-4): \quad 273-282$

Murray J E 1969 The drift, deterioration and distribution of icebergs in the North Atlantic Ocean. In Ice seminar ... sponsored by the Petroleum Society of CIM, Calgary, Alberta, 1968. Montreal,

Canadian Institute of Mining and Metallurgy: 3-18 (Special Volume 10)

Napoleoni J G P Unpublished The dynamics of iceberg drift. M.Sc. thesis, University of British Columbia, Department of Geophysics and Astronomy, 1979

Neumann G 1968 Ocean currents. Amsterdam, Elsevier 